\title{
Does the microenvironment drive different development patterns in distinctly located ampullary neoplasms?
}

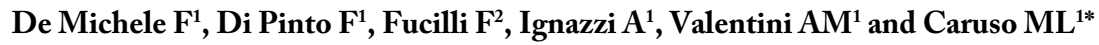 \\ ${ }^{1}$ Unit of Pathology - National Institute of Gastroenterology “S. de Bellis”, Research Hospital, Via Turi 27, Castellana Grotte, Bari, 70013, Italy \\ ${ }^{2}$ Unit of Radiology - National Institute of Gastroenterology “S. de Bellis”, Research Hospital, Via Turi 27, Castellana Grotte, Bari, 70013, Italy
}

\begin{abstract}
Tumours of the small intestine are rare and most cases of carcinoma arise in the ampullary region. We present the case of a 70-year-old patient with two synchronous lesions: a villous sessile adenoma of the papilla with a cancerous area, and a flat intraepithelial neoplasia of Vater's ampulla. The real distinction between these lesions, morphologically different and growing in two different compartments of the ampullary region, was highlighted by immunohistochemical and molecular biology results. In fact, the adenoma showed KRAS mutations while the cancer and flat dysplasia were RAS and BRAF wild type and Mismatch Repair proficient. It is possible to hypothesize: a) that the papillary and ampullary epithelium are differently stimulated by carcinogenic agents if subjected to the action of primary bile acids in the biliary tree or else exposed to the secondary bile acids in the intestinal lumen; $b$ ) that the multifactorial process of cancer driven by the diverse microbioma influences two different morphological pathways, polypoid villous adenoma and flat intraepithelial neoplasia, as occur in right and left-sided colorectal cancer development.
\end{abstract}

The presented case suggests the hypothesis that in the ampullary region different microenvironments can drive different pathways in distinctly located neoplasms.

Abbreviations: CT: Computed Tomography; EUS: echoendoscopy; MMR: mismatch repair; MSI: microsatellite instability.

\section{Introduction}

Tumours of the small intestine are rare, often associated to a malformation and most cases of carcinomas arise from the mucosa in the ampullary region [1,2]. Adenomas of the ampullary region may occur sporadically or in the context of familial polyposis and progress to malignant lesions in $4 \%$ of cases.

The ampullary region is a complex anatomical district composed of three histologically and physiologically distinct anatomic structures: the common bile duct and the main pancreatic duct converging in the papilla of Vater, and the duodenum.

The gut microbiota plays an important role in maintaining intestinal homeostasis and is particularly burdened when passing from the stomach to the small and large intestine. Particularly, the right and left-sided colon are examples of two distinct environments, coexisting in the same organ, that can differently influence genetic and epigenic changes of colonic epithelium in tumour development [3,4].

Two different environments are also recognizable in the ampullary region where the resident gut microbiota contributes to drive two different metabolically active carcinogenic pathways.

\section{Clinical summary}

We present the clinical case of a 70-year-old patient with two synchronous lesions: a villous sessile adenoma of the papilla with a cancerous area, and a flat intraepithelial neoplasia of the ampulla with high grade dysplasia.

The patient had a history of recurrent abdominal pain without obstruction and jaundice. Echoendoscopy (EUS) revealed a voluminous lobulated and pedunculated ampullary neoplasm (Figure 1).
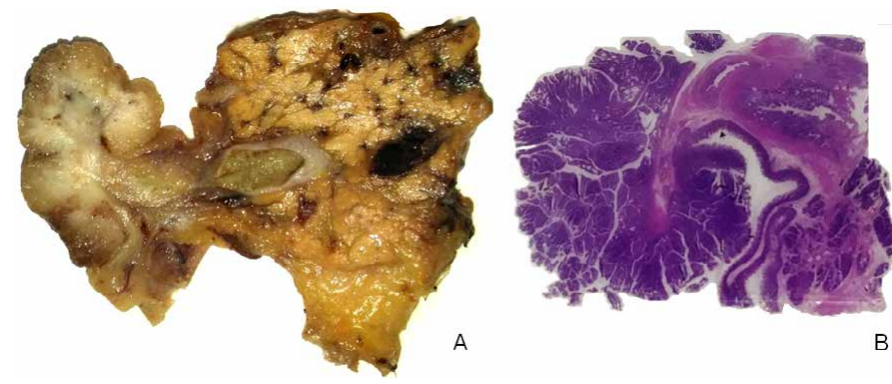

Figure 1. A) Specimen image after pancreaticoduodenectomy and B) related all mount section HE stained showing both peduncolated tubule-villous adenoma and the Vater's ampulla containing the flat intraepithelial neoplasia

The study was conducted in accordance with the ethical guidelines, and the patient gave his informed consent.

\section{Pathological finding}

On bioptic samples, histology showed a villous adenomatous lesion with severe dysplasia. Preoperative radiology revealed a $4.5 \mathrm{~cm}$ substenosing polypoid lesion not involving the ampulla and duodenal wall, without metastasis.

${ }^{\star}$ Correspondence to: Maria Lucia Caruso, Head of Pathology Unit, National Institute of Gastroenterology "S. de Bellis", Research Hospital, Via Turi 27,Castellana Grotte (Ba),70013, Italy, Tel: +39-0804994185, E-mail: mlcaruso@ irccsdebellis.it

Key words: ampullary region, flat intraepithelial neoplasia, polypoid lesion, tubule-villous adenoma, Vater's papilla

Received: November 26, 2018; Accepted: December 13, 2018; Published: December 17, 2018 
The surgical specimen was sectioned according to axial planes, a sampling technique that makes macroscopic sections anatomically equivalent to the computed tomography $(\mathrm{CT})$ images, allowing a direct comparison with the latter (Figure 2) [5]. The macroscopic sections showed a polyp with a granular and whitish area at the base and a diffuse velvety surface extending $2 \mathrm{~cm}$ into the ampulla.

Histologically, a pedunculated tubulo-villous adenoma of intestinal type with severe dysplasia was shown, and mucinous cancer limited to the mucosa and submucosa not involving the duodenal muscular layer. Furthermore, in the velvety area of the ampulla, a flat intraepithelial neoplasia of intestinal type was found, with severe dysplasia. A total of 34 lymph nodes was sampled: 4 were metastatic.

Immunohistochemical staining for p53, CK7, CK19, CK 20, SATB2, CDX2, Mismatch Repair (MMR) protein and $\beta$-catenin was performed. From the same blocks KRAS, NRAS and BRAF status was assayed by pyrosequencing. All three lesions were positive for p53, CK20, CDX2 and SATB2 while CK7 and $\beta$-catenin did not show any staining. The strongest positive staining on the flat intraepithelial neoplasia was obtained with CK19. The papillary adenoma resulted mutated at codon 12 of the KRAS gene (p.G12S) while the cancer area and the flat dysplasia were RAS and BRAF wild- type and MMR proficient.

\section{Discussion}

Ampullary adenomas can grow anywhere in the ampullary region and are often found incidentally during upper endoscopy or radiology. A preoperative diagnosis can obtained by echoendoscopy and biopsy although as in the large bowel, where endoscopic polypectomy is feasible, biopsy must not be employed for diagnosis. In ampullary polyps, too, preoperative biopsy has a high sensitivity in confirming the presence of adenoma but a poor sensitivity for adenocarcinoma, that is not diagnosed in $30 \%$ of cases [6]. Therefore, the biopsy diagnosis of adenoma does not exclude a carcinoma at the final pathology examination.

In our case, radiological diagnosis identified neither the cancerization of the polyp nor lymph nodes metastasis and histology of the biopsy did not identify the cancer.

The surgical options for ampullary tumours are standard pancreaticoduodenectomy or local excision with endoscopic ampullectomy. The latter, a less invasive treatment with less morbidity, can be the first line choice in patients with an ampullary adenoma smaller than $5 \mathrm{~cm}$ without clinical, endoscopic and radiological signs of malignancy [7]. In our case, considering the presence of the cancer and the lymph nodes metastasis, the surgical approach was the most appropriate, also in view of the size.

Ampullary polypoid tumours can be of intestinal or pancreaticobiliary type. The former stain positive to CK7, CK20 and intestinal differentiation markers such as CDX2 and SATB2 while the pancreaticobiliary type (morphologically similar to Intraductal Papillary Mucinous Neoplasms of the pancreatic bile ducts) expresses CK7 and CK17 $[8,9]$.

The role of the gut microbiota in intestinal disease changes when passing from the stomach to the colorectum. The extremely acidic gastric $\mathrm{pH}$ is a hostile environment for bacteria while the progressive increase of bacteria contents develops from the duodenum to the small and large bowel (passing from $10^{2}$ bacteria cells /g to $10^{12}$ bacteria cells $/ g$ of tissue) with a positive gradient from the proximal to the distal colon.

In addition, bile acid exposure, short - chain fatty acids and mutagenic metabolites also reach significantly higher levels in the distal colorectum where the higher concentration microbioma is related to an immune tolerance as compared to the immunogenicity on the right side $[3,10]$.

In colorectal tumorigenesis, major groups of genetic abnormality are characterized by inactivation of the APC gene and microsatellite instability (MSI). Furthermore, genes of the RAS family are found to be activated by missense mutations in $45 \%$ of colorectal neoplasias and for the serrated pathway, characterized by the BRAF oncogene mutation, the resulting carcinomas are usually MSI and are more frequently proximal $[4,11,12]$.

The KRAS oncogene mutation and p53 tumor suppressor gene are both well recognized genetic aberrations in the classical adenomacarcinoma sequence but are less documented in the development pathway of flat adenomas, where microsatellite instability was reported to be infrequent, as in our case showing MMR proficiency $[10,13,14]$.

A prevalence of flat colonic adenomas has been identified in families with hereditary non- polyposis colorectal cancer in the right side of the colon, while the protruded type is more frequent on the left $[15,16]$.

Flat adenomas have a lower incidence of major genetic abnormalities than classical protruded lesions in the adenoma- carcinoma sequence.
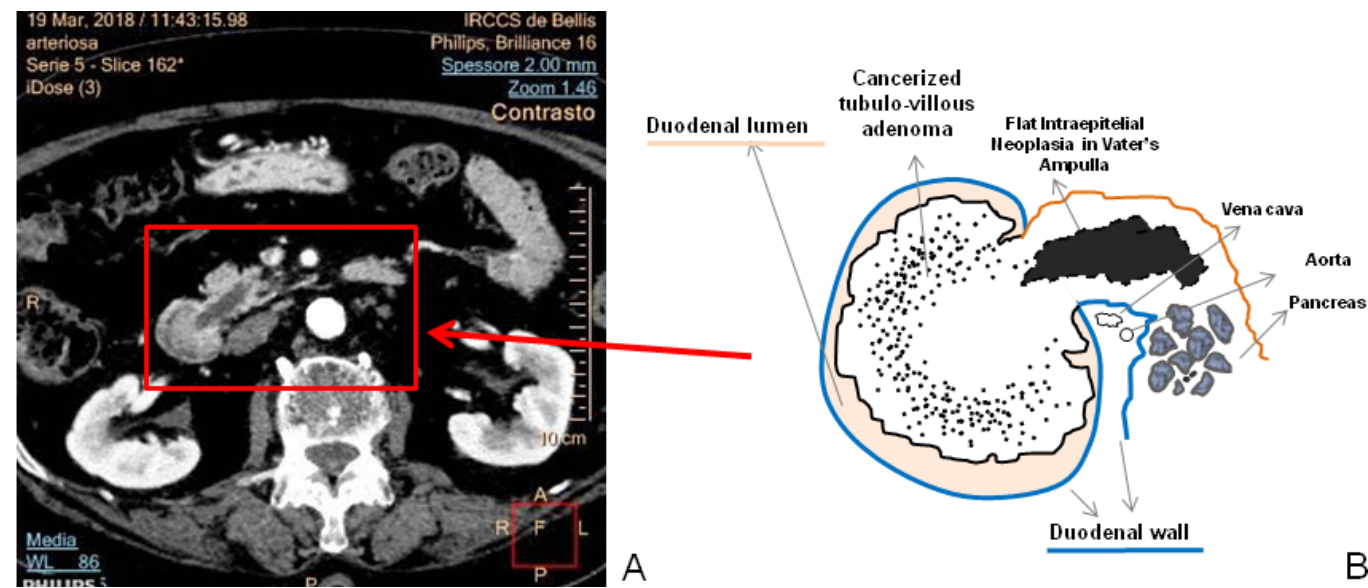

Figure 2. A) The arterial phase of CT multiphase exam with iodine contrast medium showing the tubule-villous adenoma developed into the duodenal lumen and the dilatation of main pancreatic duct and B) the related drafted illustration 
The most significant feature of flat lesions is that they complete the adenoma - carcinoma sequence along the alternative pathway more rapidly than occurs in left-sided colorectal tumorigenesis [17].

Thus, the left and right colon are two distinct environments that can drive the development of different phenotypic and molecular pathways: the non-polypoid (right) and the polypoid (left). Biliary and pancreatic secretions are collected and drained through the papilla of Vater into the duodenal lumen; where conjugated bile acids are deconjugated by bacteria to produce secondary bile acids. These latter, in classic mutagenesis tests, are heavily implicated in intestinal carcinogenesis and their action can enhance mutagenesis through numerous mechanisms $[18,19]$.

As occurs in the right and left colon, in the ampullary region the papilla defines the anatomical separation of two microenvironments: the intestinal lumen and the ampulla; the differences are also related to the resident microbiota, and epithelial bile acids exposure.

The case presented describes an infrequent association of two synchronous lesions: a papillary villous adenoma with a cancerous area and a flat ampullary intraepithelial neoplasia. The two lesions were in anatomical and biological continuity: both have the same intestinal phenotype, highlighted by immunohistochemical positivity but they grew according to two different morphologic patterns.

The KRAS mutation, detected in 30- $40 \%$ of patients with adenoma - carcinoma of the ampullary region, was present only in the microdissected adenomatous tissue, while the flat adenomatous neoplasia and the cancer area were wild type for the KRAS and BRAF genes [20]. This result was to be expected, but further indicates that the two lesions are different and may have undergone different pathogenic stimuli which influenced their growth, conditioning not only a distinct morphology but also a specific molecular development pathway.

As colorectal cancer exhibits differences in incidence, pathogenesis, molecular pathways and outcome on the right or left side, the presented case suggests that in the ampullary region different microenvironments can drive different pathways in distinctly located neoplasms. It is possible to hypothesize that, the papillary and ampullary epithelium are differently stimulated by carcinogenic agents when subjected to the action of primary bile acids in the biliary tree or when exposed to the secondary bile acids in the intestinal lumen with the contribution of the resident microbioma, influencing two different morphological pathways: a polypoid villous adenoma and a flat intraepithelial neoplasia, supported by different genetic abnormalities.

\section{Authors contributions}

F.D.M. and M.L.C. conceived and designed the study; F.D.P. and F.F. acquired and analyzed data; F.D.M., M.L.C. and A.I. drafted the manuscript and the figures, A.M.V. performed immunohistochemistry and molecular biology. All authors read and approved the final manuscript.

\section{Funding information}

The authors were not funded for this work.

\section{Competing interest}

The authors declare that they have no competing interests.

\section{References}

1. Caruso ML, Marzullo F (1988) Jejunal adenocarcinoma in congenital heterotopic gastric mucosa. J Clin Gastroenterol 10: 92-94. [Crossref]
2. Caruso ML, Cavalcanti E, De Michele F, Ignazzi A, Carullo R, et al. (2018) Small bowel capsule endoscopy revealing neuromuscular and vascular hamartoma of the jejunum: A case report. Medicine 97: e0196. [Crossref]

3. Lee GH, Malietzis G, Askari A, Bernardo D, Al-Hassi HO, et al. (2015) Is right-sided colon cancer different to left-sided colorectal cancer? - a systematic review. Eur J Surg Oncol 41: 300-8. [Crossref]

4. Breivik J, Lothe RA, Meling GI, Rognum TO, Borresen-Dale AL, et al. (1997) Different genetic pathways to proximal and distal colorectal cancer influenced by sexrelated factors. Int J Cancer 74: 664-669. [Crossref]

5. Campbell F, Verbeke CS (2013) Pathology of the pancreas. A practical approach Springer-Verlag London 29-30.

6. Elek G, Gyori S, Toth B, Pap A (2003) Histological evaluation of preoperative biopsies from ampulla vateri. Pathol Oncol Res 9: 32-41. [Crossref]

7. Onkendi EO, Naik ND, Rosedahl JK, Harmsen SW, Gostout CJ, et al. (2014) Adenomas of the ampulla of Vater: a comparison of outcomes of operative and endoscopic resections. J Gastrointest Surg 18: 1588-1596. [Crossref]

8. Furukawa T, Kloppel G, Volkan Adsay N, Albores-Saavedra J, Fukushima N, et al (2005) Classification of types of intraductal papillary-mucinous neoplasm of the pancreas: a consensus study. Virchows Arch 447: 794-799. [Crossref]

9. Berg KB, Schaeffer DF (2017) SATB2 as an immunohistochemical marker for colorectal adenocarcinoma: A concise review of benefits and pitfalls. Arch Pathol Lab Med 141: 1428-1433. [Crossref]

10. Rubio CA, Rodensjo M (1996) Mutation of p53 tumor suppressor gene in flat neoplastic lesions of the colorectal mucosa. Dis Colon Rectum 39: 143-147. [Crossref]

11. Soong R, Powell B, Elsaleh H, Gnanasampanthan G, Smith DR, et al. (2000) Prognostic significance of TP53 gene mutation in 995 cases of colorectal carcinoma. Influence of tumour site, stage, adjuvant chemotherapy and type of mutation. Eur J Cancer 36: 2053-2060. [Crossref]

12. Nosho K, Irahara N, Shima K, Kure S, Kirkner GJ, et al. (2008) Comprehensive biostatistical analysis of $\mathrm{CpG}$ island methylator phenotype in colorectal cancer using a large population-based sample. PLoS One 3: e3698.

13. Higashidani Y, Tamura S, Morita T, Tadokoro T, Yokoyama Y, et al. (2003) Analysis of $\mathrm{K}$-ras codon 12 mutation in flat and nodular variants of serrated adenoma in the colon. Dis Colon Rectum 46: 327-332. [Crossref]

14. Kinney TP, Merel N, Hart J, Joseph L, Waxman I (2005) Microsatellite analysis of sporadic flat and depressed lesions of the colon. Dig Dis Sci 50: 327-330. [Crossref]

15. Lynch HT, Smyrk T, Lanspa SJ, Marcus JN, Kriegler M, et al. (1988) Flat adenomas in a colon cancer-prone kindred. J Natl Cancer Inst 80: 278-282. [Crossref]

16. Cristofaro G, Lynch HT, Caruso ML, Attolini A, DiMatteo G, et al. (1987) New phenotypic aspects in a family with Lynch syndrome II. Cancer 60: 51-58. [Crossref]

17. Kudo Se, Lambert R, Allen JI, Fujii H, Fujii T, et al. (2008) Nonpolypoid neoplastic lesions of the colorectal mucosa. Gastrointest Endosc 68: S3-47.

18. Gill CI, Rowland IR (2002) Diet and cancer: assessing the risk. Br J Nutr 88 Suppl 1 S73-S87. [Crossref]

19. Kawalek JC, Hallmark RK, Andrews AW (1983) Effect of lithocholic acid on the mutagenicity of some substituted aromatic amines. J Natl Cancer Inst 71: 293-298. [Crossref]

20. Schultz NA, Roslind A, Christensen IJ, Horn T, Hogdall E, et al. (2012) Frequencies and prognostic role of KRAS and BRAF mutations in patients with localized pancreatic and ampullary adenocarcinomas. Pancreas 41: 759-766. [Crossref]

Copyright: (C2018 De Michele F. This is an open-access article distributed under the terms of the Creative Commons Attribution License, which permits unrestricted use, distribution, and reproduction in any medium, provided the original author and source are credited. 